\title{
Psychometric properties of the German Version of the Psychological Consequences of Screening Questionnaire (PCQ)
}

Urs Alexander Fichtner ( $\boldsymbol{\sim}$ Urs.Fichtner@uniklinik-freiburg.de)

Universitätsklinikum Freiburg: Universitatsklinikum Freiburg https://orcid.org/0000-0002-4190-8969

Andy Maun

Universitatsklinikum Freiburg

Erik Farin-Glattacker

University Medical Center Freiburg: Universitatsklinikum Freiburg

\section{Research}

Keywords: Psychological, liver diseases, non-cancer setting, internal consistency, scale structure, convergent validity

Posted Date: December 4th, 2020

DOl: https://doi.org/10.21203/rs.3.rs-120430/v1

License: (c) (i) This work is licensed under a Creative Commons Attribution 4.0 International License. Read Full License 


\section{Abstract \\ Background:}

This study aimed to translate the negative and positive items of the Psychological Consequences Questionnaire (PCQ) into German, to adapt this version to the context of screening for cirrhosis of the liver, and to test its psychometric properties.

\section{Methods:}

The three subscales (physical, emotional, and social) were translated into German using a forwardbackward translation method. Furthermore, we adapted the wording to the context of liver diseases. We tested the acceptability, distribution properties, internal consistency, scale structure, and the convergent validity using an analysis sample of 443 patients who were screened for cirrhosis or fibrosis of the liver.

\section{Results:}

We found low non-response and non-unique answer rates on the PCQ items in general. However, positive items had higher non-response rates. All items showed strong floor effects. Cronbach's alpha was high for both the negative $(a=0.95)$ and the positive PCQ scale $(a=0.90)$, as well as for the total PCQ scale ( $a$ $=0.90$ ). . Confirmatory factor analysis could reproduce the three dimensions that the PCQ intends to measure. However, it suggests not summing up a total PCQ score and instead treat the subscales separately. Convergent validity with the short form of the Spielberger State-Trait Anxiety Inventory (STAIY-6) was acceptable.

\section{Conclusions:}

Overall, our study results report a successful adaptation of the German PCQ with good performance in terms of acceptability, internal consistency, scale structure, and convergent validity. We could demonstrate that the German version of the PCQ is a useful and well-performing measurement for both negative and positive screening consequences, even in a non-cancer setting.

\section{Background}

Medical screening is increasingly used in any medical discipline to detect the onset of diseases and prevent severe progression. Thus, screenings are commonly used in a population that has no acute symptoms. In Germany, a screening procedure called "Gesundheits-Check-Up" (health check-up) that aims to detect risk factors for certain diseases is available for statutory health insurance members aged 35 and higher and includes a blood and urine test [1]. A newly introduced screening procedure for the early detection of cirrhosis or fibrosis of the liver is tested in the context of the ongoing SEAL programme 
(SEAL - Structured early detection of asymptomatic cirrhosis of the liver in Rhineland-Palatia and Saarland) in two German Federal States (Rhineland-Palatia and Saarland) since January 2017 [2]. This programme integrates the screening in the "Gesundheits-Check-Up" routine which can be repeated every three years.

Beside the benefits of early detection of diseases, such as early treatment and potential prevention, negative effects should also be taken into consideration when evaluating the role of screenings [1, 3]. In a review by Harris [3] potential harms that cross multiple conditions, e.g. nonadherence, overdiagnosis and targeted screening are discussed. Landstra et al. [4] contribute to this discussion by outlining that unrealistic optimism caused by negative screening results can lead to lower anxiety and thus to a reduction in health-promoting behaviours. A dilemma that occurs when introducing broad screening programmes is that they tend to have low predictive power resulting in a high rate of false-positive results. Studies have shown that false-positive screening results have substantial negative psychosocial consequences [5, 7-9]. However, not only false-positive results can have that impact. Intensive surveillance during the screening process itself can produce unfavourable side effects on psychological well-being and health-related quality of life due to the confrontation with a potential threat [6]. In order to identify potential burden in line with the screening, we conducted a cross-sectional survey alongside the SEAL study with screened participants.

Brodersen et al. [5] emphasize the need for patient-reported outcome measures (PRO) with high content validity in order to systematically investigate psychosocial consequences of screening. To the best of our knowledge, no instrument exists so far to measure the psychological impact of screening for liver diseases, particularly not in German language. Therefore, we chose to adapt a questionnaire that was initially developed in the context of breast cancer screening. The Psychological Consequences Questionnaire (PCQ) was created 1992 in Australia and aims at measuring the positive and negative effects of breast cancer screening on emotional, physical and social functioning [7]. To date, the PCQ was broadly used in measuring short-term screening impact in the context of mammography screening [6-11] and was also adapted to other types of cancer such as colorectal [12], anal [13] and skin cancer [14]. Cross-cultural adaptations produced translated versions of the PCQ in Dutch [6] and Danish [15] indicating a high usability of the instrument. To best of our knowledge, no German version of the PCQ exists so far. We also found no attempt for an adaptation of the PCQ beyond cancer diseases in the literature. However, we assume that liver cirrhosis is comparable to cancer in terms of the life-threatening perception of the population and thus consider the adaptation of the PCQ as suitable in this context. Consistent with the translation into Danish [15], we adapted not only the negative but also the positive items of the PCQ. The original PCQ comprises the three emotional, psychological and social dimensions, both measured by negative and positive items. Emotional consequences are measured by five negative items and five positive items. Four negative and three positive items measure the psychological aspects and three negative and two positive items picture the social dimension (see Table 1). All items were rated on a 4-point scale ranging from 0 to 3 . The exact scale labels differed slightly between negative and positive items and were 0 "not at all", 1 "rarely" and "a little bit", 2 "some of the time" and "quite a bit" and 3 "quite a lot of the time" and "a great deal". They can be added to scores for each dimension after 
recoding the positive consequences to express the level of dysfunction. Along with suggestions in the literature, we tested an overall PCQ score as a single concept of adverse psychological consequences as well $[6,8]$.

The purpose of this study is threefold:

1. to translate the PCQ into German

2. to adapt it to the context of liver diseases

3. to assess acceptability, internal consistency, scale structure and validity of the questionnaire in a screening population

\section{Methods}

\section{German version of the PCQ}

In a first step, we slightly rephrased the items to fit to the context of liver screening. We then applied forward and backward translation procedures for all PCQ items [16]. First, two German native speakers with fluent command of English and social science background independently translated the PCQ into German and found consent on their versions. Second, an English native speaker with fluent command of German and a bilingual professional translator translated the German text back into English. After minor revisions, the team found consent on a German version which was further discussed in a group of German native speaking Social Scientists $(n=5)$ with expertise in survey design.

\section{Study population and data collection}

The ongoing SEAL study is a prospective study that aims at evaluating a newly introduced medical screening method for early diagnosis of cirrhosis or fibrosis of the liver. Since May 2018, patients who visit collaborating clinics or doctor's offices in Rhineland-Palatia or Saarland for a check-up were screened for liver cirrhosis and fibrosis. The screening process itself is a multistep design depending on the test result of each step (step 1: blood sample test and risk score, step 2: enhanced laboratory diagnostics and ultrasound, step 3: liver biopsy and enhanced diagnostics in a specialized clinic. Inclusion criteria for study participation were a minimum age of 35 and no known previous cirrhosis of liver [2].

In August 2019, we contacted all patients who were included in the study so far. Due to ethical considerations, it was not possible to assess information on the phase in which the patients were in the whole screening process. This means that we could not gather information on whether patients with positive screening results already moved to step 2 or 3 or whether they still wait for an appointment. Therefore we could not control for false-positive results and potential effects caused by them. Since screening began in May 2018, we assume that the majority of the patients already received information on the test results. However, previous qualitative interviews revealed that patients, in general, receive no information in case of negative test results (results that showed no pathological findings) at all. A gross 
sample of 5.935 patients received a postal mail including a self-administered questionnaire, patient information and informed consent. With a return rate of $9 \%$ in those who were negatively screened and a return rate of $12 \%$ in those who were positively screened, we received 499 (negatively screened) respectively 21 (positively screened) completed questionnaires. In some cases, signed informed consent for our survey was missing. After a subsequent acquisition of missing consent documents, we excluded those cases that were not legitimate for evaluation $(n=34)$. We ended up with a net sample of 487 patient questionnaires $(n=19$ positively screened and $n=468$ negatively screened). For this analysis, we included only cases with at least an $80 \%$ response rate to the PCQ items. This means we excluded cases that had more than three missing items on the negative PCQ scales and more than two missing responses on the positive PCQ scales. To increase comparability, we followed the approach of Rijnsburger et al. and imputed median scores per item in eligible questionnaires [6]. Furthermore, in case of non-unique answers, we treated those as missing values. For psychometric analysis we ended up in an analysis sample of 443 cases.

\section{Statistical Analyses}

For cross-cultural comparability, the analyses follow the suggestions of Rijnsburger et al. [6]. However, we slightly extended our psychometric test procedures according to Schupp et al. [17] to reach a better understanding of the psychometric properties of the PCQ. The analyses were conducted using IBM SPSS Statistics 26.

\section{Distribution Properties}

To give insights into the distribution properties, we computed skewness and kurtosis. Non-response rates and double cross rates (non-unique answers) for each item were shown to understand acceptability of the scale. Additionally, items with high skewness or kurtosis as well as items that show ceiling or floor effects were determined. We followed the classification for ceiling and floor effects as suggested by McHorney \& Tarlov, Varni et al. and Lin et al. which suggests a percentage of 0 to $15 \%$ as small, 16 to $30 \%$ as moderate and more than $30 \%$ as substantial floor or ceiling effect [18-20]. Furthermore, we showed the 25th, 50th and 75th percentiles of the subscales and the total PCQ.

\section{Internal consistency}

We computed Cronbach's Alpha for the PCQ scales to evaluate internal consistency. We also assessed item-total correlation as well as mean-inter-item correlation for each subscale. Furthermore, we tested whether Cronbach's Alpha allows computing an overall PCQ including both negative and (recoded) positive items.

\section{Scale structure}

We conducted a confirmatory factor analysis (CFA) using IBM SPSS AMOS 24 software to confirm the item-factor-relationship. Since the PCQ is assumed to cover three dimensions, a three-factor solution was tested based on Maximum Likelihood estimation. Model fit was evaluated using $\mathrm{Chi}^{2}$ goodness of fit test, the Comparative Fit Index (CFI) [21], Tucker-Lewis Index (TLI) [22], root mean square error of 
approximation (RMSEA) and standardized root mean square residual (SRMR). A non-significant $\mathrm{Chi}^{2}$ test indicates that the assumed model fits the data well. We consider CFI and TLI values above 0.90 as an indication of a good fit. RMSEA values below 0.1 are considered as moderate and values below 0.05 as good fit [19].

\section{Convergent validity}

To evaluate convergent validity, we correlated the PCQ subscales with the Spielberger State-Trait Anxiety Inventory (STAI Short version Y-6) [23]. This scale is designed to measure current emotional status and is assumed to correlate high (Pearson's r greater than 0.5) with all three subscales of the PCQ. We further hypothesize that the highest correlation to be found is with the emotional subscale of the PCQ. If this hypothesis cannot be rejected, we conclude acceptable convergent validity of the German version of the PCQ. Additionally, we assume that the STAI-Y- 6 scale correlates stronger with the negative PCQ subscales than with the (recoded) positive PCQ subscales. This assumption is based on the rationale that the absence of a positive effect (e.g. not experiencing greater well-being) should not be considered as equal to the occurrence of a negative effect. This circumstance led Brodersen \& Thorsen to rephrasing the positive items, so that they allow changes in both directions [15].

\section{Results}

\section{Acceptability}

We noticed low unit-non response for all PCQ items in our sample. Following the approach explained above, we had to exclude 15 cases for the negative PCQ items and 66 cases for the positive PCQ items since they show less than $80 \%$ of overall scale response. This pattern illustrates that the general nonresponse rate is higher for the positive PCQ items (ranging from 3.3-8.8\%) than for the negative items (ranging from 2.3-3.9\%) (see Table 1). We could not identify any non-response pattern that is associated with a specific subscale. Non-unique answers were generally low and were not observed more than three times per item (highest non-unique answer rate of $0.6 \%$ in item "feeling more able to do things which I normally did before").

\section{Distribution properties}

For all negative PCQ items we found mean values ranging between the categories " 0 Not at all" and " 1 Rarely" indicating that the impact of screening is generally low. This pattern also explains a substantial floor effect for all negative items with more than $50 \%$ of responses lying in the undermost category. In contrast, we found small ceiling effects with the highest percentage of values in the item "I felt worried about my future". As consequence, the data is highly skewed. Z-standardised skewness exceeds the cutoff value of +1.95 for any negative PCQ item. Regarding kurtosis, items 2, 4, 6, 9, 10,11 and 12 lie beyond the range of -1.95 to +1.95 indicating no normal distribution.

The positive PCQ items generally show higher mean values ranging from 0.88 to 2.01 indicating a tendency towards a higher item agreement to positive effects of the screening. This pattern is in line with 
fewer floor effects for the positive items in comparison to the negative items, so that substantial floor effects were found for item 13, 14, 15, 21 and 22, moderate effects were found for items 18, 19 and 20 and small floor effects were found for items 16 and 17. Substantial ceiling effects were only found for item 16, apart from that, the ceiling effects for the positive PCQ scales can be considered as small to moderate in general. Regarding skewness and kurtosis, the positive items show values closer to zero than the negative items. However, most of them exceed the cut-off values $+/-1.96$, indicating no normal distribution of the data. One should notice that all positive PCQ items show negative signs for Kurtosis.

\section{Internal consistency}

For this section and the subsequent analyses, we applied missing value imputation as stated above. Furthermore, we recoded the positive items to compute the subscale indices so that higher scale values indicate less positive impact. In general, we found acceptable Cronbach's alpha higher than 0.7, except for the overall social subscale $(a=0.58)$ (see Table 2 ). For the physical and the social subscale higher Cronbach's alpha values can be found if they were treated separately (positive and negative subscale). For the emotional dimension we found a small decrease for the overall scale compared the separate scales, which, however, still shows high reliability $(a=0.84)$. This pattern is also reflected in the mean inter-item correlation. In general, the overall subscales show lower values than if treated separately. Regarding the total PCQ scales (positive, negative, total), we found excellent Cronbach's alpha values of 0.9 and higher indicating good consistency. For the negative subscales and the total negative PCQ scale, we found strong deviation between the scale mean and the median indicating that the majority of our sample is not affected strongly in general, but bias by few extreme values occurs.

\section{Scale structure}

Table 3 shows the model fit statistics for three confirmatory factor analyses we computed. The first row represents the results for the negative PCQ model (all three negative subscales included), the second row for the positive PCQ model (all three positive subscales included) and the third row for the total PCQ model (all subscales included). For each CFA model, we conducted a modification based on the suggestions by modification indices and whether they were theoretically plausible. In general, no model showed a non-significant $\mathrm{Chi}^{2}$ test. However, it is considered too sensitive for large sample sizes [24] and we could at least reach a decrease of $50 \%$ respectively $66 \%$ in $\mathrm{Chi}^{2}$ value by modification. In all three models, we could improve the model fit expressed in an increase of the TLI and CFI as well as a decrease in RMSEA. While the modified CFA model for the negative PCQ subscales (TLI $=0.97, \mathrm{CFI}=0.98, \mathrm{RMSEA}=$ 0.07 ) and the modified CFA model for positive PCQ subscales $(T L I=0.95, C F I=0.97$, RMSEA $=0.08$ ) show quite good model fit, the total PCQ model performs badly even after modification. A closer look at the modification indices led to the assumption that some items are not selective enough and correlate with multiple concepts. Modification indices suggested a correlation between error terms of items 10 and 11 (physical subscale) with item 9 (social subscale) and the emotional subscale itself. Item 10 refers to difficulties doing things at home and item 11 refers to difficulties meeting work or other commitments. Item 9 refers to withdrawing from close people reflecting a dimension of social isolation. What those entire items share in common is that they are typical symptoms of depression. We consider that as a 
prime example of the bio-psycho-social model [25] which indicates the difficulty of separating those three dimensions from each other.

\section{Convergent validity}

As exposed in Table 4, we have to reject our hypotheses partially. Only the emotional subscale shows a strong correlation $(r=0.53)$ with the STAI-Y-6. The physical and social overall subscales show moderate positive correlations with the STAI-Y- 6 ranging from 0.40 to 0.42 . However, the total PCQ scale fits the hypothesis so that a strong correlation of 0.52 can be found. As we assumed, all positive subscales correlate less strongly with the STAI-Y- 6 than the negative subscales do. Since we also found evidence for the assumption that the emotional subscale is stronger related to the STAI-Y- 6 than the other subscales are, we overall can assume acceptable convergent validity of the PCQ.

\section{Discussion}

Overall, our study results report a successful adaptation of the German PCQ with good performance in terms of acceptability, internal consistency, scale structure and convergent validity. The non-response rates and non-unique answer rates were negligible. Our results were comparable to other adaptations of the PCQ $[6,15]$ and demonstrate that the PCQ is not only useful for the setting of cancer diseases.

However, we found substantial floor effects, especially for the negative PCQ items. Floor effects pose a potential risk in terms of accuracy of a scale since one has to assume that some kind of variation is drawn together in the lowest category. In general, we would suggest further differentiating the response categories, but this would also have as consequence that homogeneity of measurement across studies and countries would suffer. Since the lowest category is "not at all" one can assume that the PCQ produces floor effects if the population simply does not experience the eligible dysfunction. Here, it seems that the overall psychosocial impact of the screening is quite low.

Reliability analyses suggest not summing up the physical, social and emotional subscales for positive and negative items. Moreover, the separation of the two scales should be applied as intended by Cockburn et al. and confirmed by Ong et al. into a negative and a positive subscale $[7,8]$.

Because of the cut-off value of $80 \%$ which we chose as a criterion for too incomplete scales for analysis, we had to exclude 29 cases only because of the positive subscales and four cases only because of the negative items. In line with a generally higher non-response rate of the positive items, we assume that here fatigue effects might have occurred. The positive items were placed as a block after the negative items in our questionnaire at the end of the page. Since our study could not apply randomization of items, we recommend further testing of the German version of the PCQ including tests for order effects.

Another limitation of our study design was that we could not contact the patients in a specific period after their screening so that some patients have longer periods between the screening experience and responding to the questionnaire than other ones. An individual contacting procedure (e.g. 4 weeks after each test) would improve the comparability of the measurement, but was not realistic to implement in 
this study. We recommend further testing the German PCQ in a controlled setting, which could also offer the possibility to get insights into retest reliability and the sensitivity of measuring changes over time. This could shed light on the progress of potential burden after a screening experience.

The general low response rate to our study limits representativeness of our results. After the delivery of the questionnaire, we received some phone calls of patients who were not aware of their inclusion into this study. Cognitive impairment and lingual problems also were named as reasons for non-response to our questionnaires.

Due to the weak performance of the modified CFA model for the total PCQ and because of the shared variance of items 9, 10,11 and the emotional subscale, a pattern that occurs only in the total PCQ model, we recommend being careful in using the total PCQ score and instead computing the negative and the positive PCQ separately.

Since the negative PCQ subscale and the total PCQ correlated at least moderately with the STAI-Y- 6 , we consider the convergent validity as acceptable. However, more insights into other aspects of validity would help to further evaluate the performance of the PCQ. In other validation studies, the Impact of Event Scale (IES) and the Hospital Anxiety and Depression Scale (HADS) were used for validation. In our project, the IES seemed not to be adequate since most of our participants were screened negatively. Thus the considered event "liver cirrhosis" is not as present as it is in the context of the breast cancer screening study of Rijnsburger et al. [6]. The HADS was also not considered to be useful in our context since it contains a longer list of items than the short form of the STAI. However, for the future implementation of the PCQ we recommend also considering the HADS or IES to enhance international comparability of the German version of the PCQ.

\section{Conclusions}

Overall, our study results report a successful adaptation of the German PCQ with good performance in terms of acceptability, internal consistency, scale structure, and convergent validity. We could demonstrate that the German version of the PCQ is a useful and well-performing measurement for both negative and positive screening consequences, even in a non-cancer setting.

\section{Abbreviations}

PCQ - Psychological Consequences Questionnaire

STAI-Y-6 - Spielberger State-Trait Anxiety Inventory

SEAL - Structured early detection of asymptomatic cirrhosis of the liver in Rhineland-Palatia and Saarland PRO - Patient-Reported Outcomes CFI - Comparative Fit Index 
TLI - Tucker-Lewis Index

RMSEA - root mean square error of approximation

SRMR - standardized root mean square residual

CFA - confirmatory factor analysis

IES - Impact of Event Scale

HADS - Hospital Anxiety and Depression Scale

\section{Declarations}

\section{Ethics approval}

This study was performed in line with the principles of the Declaration of Helsinki. The study has been granted by the Ethics Committees of Rhineland-Palatinate and Saarland. Furthermore, it was reviewed and approved by the data security officer of the Medical Center of the University of Freiburg. Data collection was based on written informed consent. In case of missing informed consent, we excluded those cases from analysis.

\section{Consent for publication}

Not applicable

\section{Availability of data and material}

Due to ethical considerations the data cannot be published. The German version of the PCQ is available on request from the authors. The source code for the analysis is written in SPSS and can be requested of the corresponding author.

\section{Competing interests}

The authors declare that they have no conflict of interest.

\section{Funding}

The data was collected within a project funded by the Innovation Fund of Federal Joint Committee Gemeinsamer Bundesausschuss (G-BA) Germany. Funding ID: 01 NVF16026

\section{Authors' Contributions}

The first author (UF) wrote the draft of the manuscript and was responsible for survey design, data collection and analysis. The second (AM) and third author (EF) reviewed the manuscript and revised it critically. The third author was project leader and mentored the development of the questionnaire used in 
the project. The first and the third author translated the questionnaire into German and reconciled their versions. All authors approved the manuscript and gave consent to submission.

\section{Acknowledgements}

We thank the colleagues of Jill Cockburn for granting us substitutional to use and adapt the PCQ into the German context. Furthermore we want to thank Derek Hazard, Rieka von der Warth and Carole Cürten for guiding the translation process. We also thank the SEAL consortium for enabling us to conduct this study within the SEAL project. We would like to acknowledge Isabelle Hempler, Sabrina Lorenz and Martin Treppner for providing creative feedback on this article. Finally we would like to thank all the patients in Rhineland-Palatia and Saarland who gave their time to participate in the study.

\section{Licensing}

We contacted the authors of the original publication on the development of the instrument. Since Jill Cockburn as the first author deceased, one of her colleagues granted us to use the questionnaire for further adaptation.

\section{References}

1 Institute for Quality and Efficiency in Health Care - IQWiG Germany. Benefits and risks of Screening Tests. 2019. www.informedhealth.org. Accessed 26 October 2020.

2 Nagel M, Arslanow A, Nguyen-Tat M., Wörns MA, Heil FJ, Mainz D, Zimper G, Zwerenz B, Wangler J, Jansky M, Gisch D, Jäger J, Engelmann T, Graf E, Knaus J, Stelzer D, Fichtner U, Binder H, Farin-Glattacker E, van Ewijk R, Ortner J, Velthuis L, Lammert F, Galle PR. Lebervorsorge-Progamm SEAL: Screening der Allgemeinbevölkerung zur strukturierten Früherkennung von Leberfibrose und Leberzirrhose (preventive liver programme SEAL: Screening the general population for early detection of liver fibrosis and cirrhosis). Zeitschrift für Gastroenterologie. 2019; 57(09): 246.

3 Harris R. Overview of Screening: Where We Are and Where We May be Headed. Epidemiologic Reviews. 2011; 33: 1-6.

4 Landstra J, Deane FP, Ciarrochi J. Psychological consequences of cancer screening in HIV. Current Opinion in Oncology. 2013; 25(5): 526-531.

5 Brodersen J, McKenna SP, Doward LC, Thorsen H. Measuring the psychosocial consequences of screening. Health and Quality of Life Outcomes. 2007; 5: 3.

6 Rijnsburger AJ, Essink-Bot ML, van As E, Cockburn J, de Koning HJ. Measuring psychological consequences of screening: Adaptation of the Psychological Consequences Questionnaire into Dutch. Quality of Life Research. 2006; 14: 933-940. 
7 Cockburn J, De Luise T, Hurley S, Clover K. Development and validation of the PCQ: A questionnaire to measure the psychological consequences of screening mammography. Social Science and Medicine. 1992; 34: 1129-1134.

8 Ong G, Austoker J, Brett J. Breast screening: Adverse psychological consequences one month after placing women on early recall because of a diagnostic uncertainty. A multicentre study. Journal of Medical Screening. 1997; 4: 158-168.

9 Brett J, Austoker J, Ong G. Do women who undergo further investigation for breast screening suffer adverse psychological consequences? A multi-centre follow-up study comparing different breast screening result groups five months after their last breast screening appointment. Journal of Public Health Medicine. 1998; 20: 396-403.

10 Lowe JB, Balanda KP, Del Mar C, Hawes E. Psychologic distress in women with abnormal findings in mass mammography screening. Cancer. 1999; 85: 1114-1118.

11 Bowland L, Cockburn J, Cawson J, Anderson HC, Moorehead S, Kenny M. Counselling interventions to address the psychological consequences of screening mammography: A randomised trial. Patient Education and Counseling. 2003; 49: 189-198.

12 Denters MJ, Deutekom M, Essink-Bot ML, Bossuyt PM, Fockens P, Dekker E. FIT false-positives in colorectal cancer screening experience psychological distress up to 6 weeks after colonoscopy. Support Care Cancer. 2013; 21: 2809-2815.

13 Tinmouth J, Raboud J, Ali M, Malloch L, Su D, Sano M, Lytwyn A, Rourke SB, Rabeneck L, Salit I. The Psychological Impact of Being Screened for Anal Cancer in HIV-Infected Men Who Have Sex With Men. Diseases of the Colon \& Rectum. 2011; 54(3): 352-359.

14 Risica PM, Matthews NH, Dionne L, Mello J, Ferris LK, Saul M, Geller AC, Solano F, Kirkwood JM, Weinstock MA. Psychological consequences of skin cancer screening. Preventive Medical Reports. 2018; 10: 310-316.

15 Brodersen J, Thorsen H. Psychosocial consequences of false positive screening mammography - an adaptation of the Psychological Consequences Questionnaire (PCQ) into Danish. Abstract 1265. Quality of Life Research. 2003; 12: 798.

16 WHO. WHO Research tools. 2020.

https://www.who.int/substance_abuse/research_tools/translation/en/. Accessed 27 October 2020.

17 Schupp JC, Fichtner UA, Frye BC, Heyduck-Weides K, Birring SS, Windisch W, Criée CP, MüllerQuernheim J, Farin E. Psychometric properties of the German version of the Leicester Cough Questionnaire in sarcoidosis. PLoSOne. 2018; 13(10): e0205308. 
18 Varni JW, Seid M, Rode CA. The PedsQL: measurement model for the pediatric quality of life inventory. Medical Care. 1999; 37: 126-139.

19 Lin CY, Luh, WM, Cheng CP, Yang AL, Su CT, Ma HI. Measurement equivalence across child selfreports and parent-proxy reports in the Chinese version of the pediatric quality of life inventory version 4.0. Child Psychiatry and Human Development. 2013; 44: 583-590.

20 McHorney CA, Tarlov AR. Individual-patient monitoring in clinical practice: are available health status surveys adequate? Quality of Life Research. 1995; 4: 293-307.

$21 \mathrm{Hu}$ L, Bentler PM. Cutoff criteria for fit indexes in covariance structure analysis: Conventional criteria versus new alternatives. Structural Equation Model A Multidisciplinary Journal. 1999; 6: 1-55.

22 Tucker LR, Lewis C. A reliability coefficient for maximum likelihood factor analysis. Psychometrika. 1937; 38: 1-10.

23 Marteau TM, Bekker $H$. The development of a six-item short-form of the state scale of the Spielberger State-Trait Anxiety Inventory (STAI). British Journal of Clinical Psychology. 1992; 21: 301-306.

24 Chen FF. Sensitivity of Goodness of Fit Indexes to Lack of Measurement Invariance. Structural Equation Model A Multidisciplinary Journal, 2007; 14: 464-504.

25 Schotte CKW, Van Den Bossche B, De Doncker D, Claes S, Cosyns P. A biopsychosocial model as a guide for psychoeducation and treatment of depression. Depression and Anxiety. 2006; 23: 312-324.

\section{Tables}

Due to technical limitations, table 1, 2, 3, 4 is only available as a download in the Supplemental Files section.

\section{Supplementary Files}

This is a list of supplementary files associated with this preprint. Click to download.

- Table1.pdf

- Table1.pdf

- Table2.pdf

- Table2.pdf

- Table3.pdf

- Table3.pdf

- Table4.pdf

- Table4.pdf 
- STROBEchecklistPCQ.pdf

- STROBEchecklistPCQ.pdf

Page 14/14 